OPEN ACCESS

Edited by:

David John Manton,

University Medical Center

Groningen, Netherlands

Reviewed by:

Rosa Helena Wanderley Lacerda,

Federal University of Paraíba, Brazil

Mine Koruyucu,

Istanbul University, Turkey

${ }^{*}$ Correspondence:

Alexandre R. Vieira arv11@pitt.edu

Specialty section: This article was submitted to

Pediatric Dentistry,

a section of the journal

Frontiers in Dental Medicine

Received: 09 March 2021 Accepted: 04 May 2021

Published: 25 May 2021

Citation:

Vieira AR (2021) A Need for Updating the Research Agenda for Cleft Lip and

Palate and Extending the Cleft Team Model to Dental Medicine.

Front. Dent. Med. 2:678477.

doi: $10.3389 /$ fdmed.2021.678477

\section{A Need for Updating the Research Agenda for Cleft Lip and Palate and Extending the Cleft Team Model to Dental Medicine}

\author{
Alexandre R. Vieira* \\ School of Dental Medicine, University of Pittsburgh, Pittsburgh, PA, United States
}

Care for individuals born with cleft lip and palate is done by a team approach, including dental medicine. However, oral health is not integrated in other situations that affect overall health. This perspective essay makes the case for a universal team approach, having dental medicine integrated regardless of the overall health issue, much like how cleft lip and palate is managed. Furthermore, future research agenda on the etiology of cleft lip and palate in particular will need to be adjusted for a major roadblock: the lack of more sophisticated clinical descriptions for the cases ascertained at birth.

Keywords: cleft (lip and) palate, cancer, genetics, nutrition, infection, inflammation, oral bacteria

\section{INTRODUCTION}

Increasingly, the proper management of health cannot be met by a single healthcare provider, and the result has been the fragmentation of medical care among multiple consultants and services. Addressing dental needs is one of the fragmented services. Without a clearly defined manager, responsibility for the patient is often dispersed among multiple medical specialists, and not necessarily any of them has a view of the individual as a whole. Poor communication with [153mm] patients and among healthcare providers, non-continuous care, and lack of accountability further make integration more difficult $(1,2)$. To address these issues, a team approach to care has been suggested and has been particularly successful in the management of individuals born with cleft lip and palate, including an obvious role for oral health care. Therefore, the aim of this paper is to suggest that a team approach should be used in all aspects of medical care, and that this team should include a dentist. In this perspective essay, we are also reflecting on the work done in cleft lip and palate in particular. Treatment of individuals born with clefts is a model philosophy we are highlighting here as a gold standard, but the future research on cleft lip and palate may need refocus.

Aside from this contemporary approach to treat patients, it is fair to suggest that in the past 30 years, cleft lip and palate is one of the birth defects of which we have the most knowledge about its causes. There have also been great developments in the understanding of the needs of children born with cleft lip and palate. Currently, optimally treating a child born with cleft lip and palate requires coordination of multiple specialties. Nutrition $(3,4)$, psychological effects of prenatal diagnosis (5), and post-operative outcomes (6) have been evaluated, but it remains unclear which individuals may benefit more for specific interventions, approaches, and techniques. This coordination makes cleft lip and palate the perfect scenario in which dental medicine and medicine integrate, a model that would most likely benefit the treatment of other medical conditions as well. In this perspective essay, the suggested team approach to care for individuals born with cleft lip and palate is brought to light as a model that should be more widely applied to other overall health issues. However, the 
team approach to assess, plan, and care for individuals born with cleft lip and palate did not benefit from the current research effort exploring the etiology of the defect. Therefore, it is suggested that the research agenda for cleft lip and palate be adjusted to address the main gaps of its current knowledge: (i) the etiology of multifactorial cleft lip and palate, and (ii) the reasons behind the differences seen in treatment outcomes.

\section{PROPOSED FUTURE CLEFT LIP AND PALATE RESEARCH AGENDA \\ Studying the Etiology of Cleft Lip and Palate: We May Have to Start Over}

Being an obvious clinical change that can be immediately detected in the delivery room, ascertaining cases at birth became the favorite strategy to study the etiology of cleft lip and palate. Studies were designed to recruit children and their families at the time of birth, including collecting data on risk factors and exposures, and biological samples. This approach allowed for testing for associations between the phenotype and common genetic variation in the population. Heritability of cleft lip and palate has been calculated between 77 and 97\%, depending on the amount of cases that may exist that can be explained by major gene effects in relation to cases that are due to multifactorial inheritance (7). These high heritability estimates justified a focused effort on unveiling the genes that cause the defect. After the first report of an association between a common genetic variant in the population and cleft lip and palate (8), two decades of targeted work [reviewed in (9)], including family-based studies implementing hypothesis-free linkage strategies [reviewed in (10)] followed. Suddenly, three genome wide association studies were published between December 2009 and June 2010 (1113), testing the hypothesis that common genetic variations in the population that were never considered before in the targeted association work [reviewed in (9)] were associated with cleft lip and palate. A decade later, the list of genes of interest that may contribute to cleft lip and palate grew longer, but that did not lead to new proposals of preventive strategies and had no impact on how recurrence risks are calculated for the purpose of genetic counseling.

This lack of translation from the genomic work into etiologic factors motivated investigators to look at other approaches. Two strategies are worth mentioning: (i) more sophisticated clinical descriptions beyond the traditional cleft lip with or without cleft palate and cleft palate only, and (ii) detecting mechanisms that control gene expression. Cleft lip and palate appears to be accompanied with minor defects, in particular changes in dental development [reviewed in $(9,10)$ ]. Virtually, all individuals born with cleft lip and palate have dental development alterations outside the cleft area [i.e., the posterior maxillary teeth and/or mandibular teeth; (14)], and delayed dental development or tooth eruption, which implies a delay in start of orthodontic treatment (15), and it has been proposed that cleft lip and palate is always a syndrome of the dentition (16). The challenge of these assumptions, however, is that the historical cohorts recruited at birth do not have any descriptions of the dentition and recovering that information would not be a trivial task. Among the investigators around the world working on this topic, there are virtually thousands of DNA samples stored in freezers that cannot be further studied. The field is turning to epigenetic changes such as DNA methylation and histone modifications, which are key developmental mechanisms linked to the environment [reviewed in (17)]. The integration of genetic and environmental data and the study of epigenetic and other non-coding mechanisms will turn out to be relevant. Interestingly, there is already a suggestion that this work will benefit from distinguishing cleft lip and palate subtypes, and careful and sophisticated clinical descriptions will be needed, which also indicate that the historical sample cohorts may not allow for further inquiry. It is likely that further progress in the understanding of the etiology of cleft lip and palate will require redesigning protocols with more clinical detail and recruiting subjects again, including considering designs of prospective cohorts. At this time, it will be beneficial to draw from the multitude of experts that comprise a "cleft team," to include measures that are patient-centered and outcomes related to the several dimensions of treatment: nutrition, surgery, esthetics, orthodontics, oral health, speech, psychology, genetic counseling, and overall health.

\section{Outcomes of Treatment}

Variation in the results of treatment of cleft lip and palate exist but are not the focus of systematic inquiry. This is an area that deserves more attention now that safety of surgical corrective procedures is the highest ever for the children that need these treatments, and the integration of multidisciplinary care (plastic/craniofacial surgeon, pediatrician, orthodontist, pediatric dentist, speech and language specialist, otolaryngologist, audiologist, genetic counselor, nurse team coordinator, and social worker) is a concept that has evolved and is widely mentioned as being the best practice in addressing all the individual needs of people that born with clefts.

When measuring outcomes of surgery, independent of the technique or the type of cleft lip (unilateral vs. bilateral), a small subset of individuals scored poorly in measurements of vermilion roll, scar hypertrophy, cupid's bow, lip length, nostril asymmetry, alar dome, and alar base (18). A valid hypothesis is that some variation may be explained by biological mechanisms. Facial growth is another outcome of interest and the typical concave profile of individuals born with clefts is attributed to the early surgical repair, but data showed that maxillary growth may be the main determinant for the final facial profile (19), and postnatal craniofacial growth is mostly genetically determined. Wound healing is another outcome of treatment that is of interest and children with Van der Woude syndrome (altered IRF6 function) showed thicker epidermis compared with that from children with multifactorial cleft lip and palate (20). COMT rs4818 allele distribution appear to determine intensity and duration of pain post-alveolar bone graft from the iliac crest bone (21). Next generation of research on cleft lip and palate should take advantage of the team approach for treating individuals born with cleft lip and palate to focus on outcomes of all dimensions 
of treatments individuals born with cleft lip and palate undergo since birth until adulthood.

\section{THE MODEL CLEFT LIP AND PALATE TEAM APPROACH SHOULD BE EXTENDED TO ALL MEDICAL CONDITIONS}

\section{Multidisciplinary Teams}

Mechanisms for organizing and coordinating health and care services for individuals with complex care needs have been emphasized as a way to best meet the needs of these complex patients. These teams bring together the expertise and skills of several different professionals, who are expected to assess, plan, and manage cases jointly. One of the major advantages of this philosophy is that it saves patients and their families the need to have multiple appointments. It also creates an environment that is very rich, with the multiple specialties have a chance to interact in the same physical space, which enhances the professional experience and impacts positively the care of patients. Oral health is impacted by the underlying overall health and a dentist should be always a member of any team.

\section{Oral Health-Systemic Health}

One theme that was a constant in the dental literature over the last three decades includes the relevance of oral health to the overall health. Periodontitis (and in some instances dental caries and/or tooth loss) have been associated with higher mortality later in life, dementia, Alzheimer's disease, premature births, arthritis, diabetes, upper respiratory infections, pneumonia, auto-immune diseases, cardiovascular disease, obesity, and cancer [reviewed in (22)]. These associations motivated the suggestion that oral disease can cause systemic disease, and keeping a good oral health and visiting regularly one's dentist is more important than ever. Despite the positive connotation of emphasizing good oral health, the claim that oral disease causes systemic disease, possibly because oral bacteria travels through other organs does not appear to best explain the origins of systemic disease. On the contrary, it appears that poor overall health is the one directly that impacts oral health (23). A more likely explanation for the association between oral health issues, such as periodontitis, with overall health issues is that they are inflammatory in nature, and the way the host responds to inflammation is the link between these diseases. Further, it is possible to imagine that the presence of one condition worsen the other and vice-versa (24). If there was a causal relationship, treating one condition should have the effect of ameliorating the other, but that has not been seen $(25,26)$. Hence, it is the interest of the dental profession to engage in preventing deteriorating overall health of the population. Similar to the team approach proposed for treating individuals born with cleft lip and palate, in which dental medicine is explicitly represented (by pediatric dentists and orthodontists), a team approach to treat adult disease integrating a dentist would provide a solution for improving quality of life. One example in which the lack of integration of dental medicine in the setting of treating patients is treatment of cancer. An important subset of patients undergoing treatment will have oral mucositis as a side effect (100\% of cases undergoing head and neck radiotherapy for head and neck cancer, and up to $40 \%$ of patients undergoing chemotherapy for other cancer conditions) (27) $)^{1}$. Oral mucositis can be extremely painful and compromises nutrition, speech, fluid intake, and increases the risk of systemic infection. These symptoms can be so severe that patients may require hospitalization or request the treatment to be discontinued, which can have dramatic consequences on chances of survival. A dental professional present can minimize these effects by using low-level laser therapy [reviewed in (28)]. An effort should be made to integrate oral medicine specialists in hospital settings. Conversely, physicians must require that the oral cavity of the individuals with adult diseases they treat are kept healthy as part of their overall management goals.

\section{DISCUSSION}

\section{Patient Centered Outcomes Should Be on the Forefront}

When deciding outcomes of health to study, it is common that mortality is chosen to be measured, as decreased mortality is considered a benchmark for success. Patients, however, are often most concerned about pain and discomfort. Patient centered outcomes are likely to be a better measure of the impact of interventions, and if a disease has as consequence deteriorating oral health, pain, and discomfort is the result.

Patient-centered outcomes research (according to the PatientCentered Outcomes Research Institute; https://www.pcori. org/research-results/about-our-research/patient-centered-

outcomes-research) answers questions, such as:

- Given my personal characteristics, conditions, and preferences, what should I expect will happen to me?

- What are my options my options, and what are the potential benefits and harms of those options?

- What can I do to improve the outcomes that are most important to me?

- How can clinicians and the care delivery systems they work in help me make the best decisions about my health and health care?

To address these questions, study designs should assess the benefits and harms of preventive, diagnostic, therapeutic, or palliative interventions highlighting comparisons and outcomes that matter to people. Individual preferences may range from survival to function, symptoms, or quality of life issues. Study designs also should contemplate diversity, inequality, and barriers for the implementation or dissemination of interventions.

The ideal systems optimize outcomes while addressing burden to individuals, availability to services, technology and personnel challenges, and stakeholder needs. Dentists should be integrated in the care of individuals who are ill, not because it may decrease mortality, but because it improves quality of life.

${ }^{1}$ The Oral Cancer Foundation. https://oralcancerfoundation.org/complications/ mucositis 


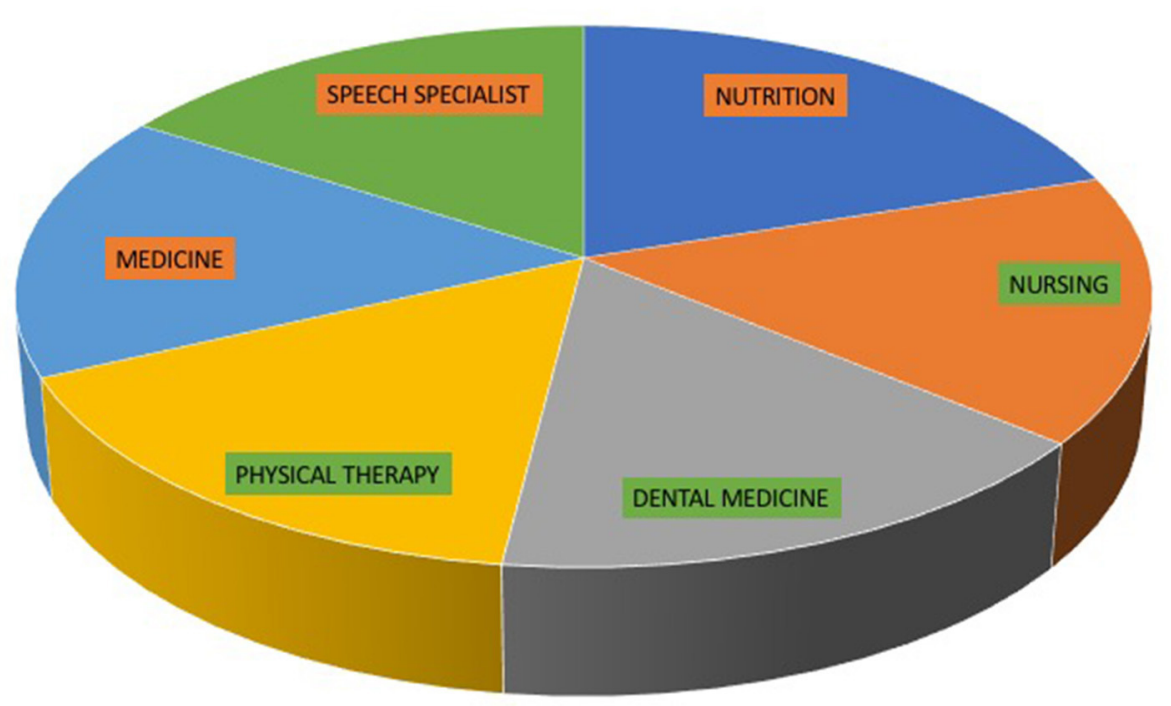

FIGURE 1 | The "team approach" that should be implemented for all overall health issues.

Certain geographic areas are promoting the presence of dental care in hospitals (often called Hospital Dentistry and even defined as a subspecialty of dental medicine with formal certification), but the ideal scenario is creating in hospital settings a sustainable team approach as seen in cleft lip and palate care (Figure 1). Future research and policy must focus on the multitude of factors that could affect the integration of dental and systemic medicine. These may include vast organizational, educational, and attitude changes to how dental medicine is practiced. Patient-centered outcomes of treatment and determination of meaningful oral and systemic health biomarkers must be emphasized. These changes will require refocusing the understanding of how health care should be provided, putting much greater thought in preventive policies, such as the taxation of cigarettes implemented last century (29). Taxation of dietary products rich in refined sugars coupled with subsidies for the production of fruits and vegetables is another intervention that will likely impact risks of the

\section{REFERENCES}

1. Stavert RR, Lott JP. The bystander effect in medical care. N Engl J Med. (2013) 368:8-9. doi: 10.1056/NELMp1210501

2. Manser T. Teamwork and patient safety in dynamic domains of healthcare: a review of the literature. Acta Anaesthesiol Scand. (2009) 53:14351. doi: 10.1111.j.1399-6576.2008.01717.x

3. Glenny AM, Hooper L, Shaw WC, Reilly S, Kasem S, Reid J. Feeding interventions for growth and development in infants with cleft lip, cleft palate or cleft lip and palate. Cochrane Database Syst Rev. (2004) 3:CD003315. doi: 10.1002/14651858.CD003315.pub2

4. Duarte GA, Ramos RB, Cardoso MCAF. Feeding methods for children with cleft lip and/or palate: a systematic review. Braz J Otorhinolaryngol. (2016) 82:602-9. doi: 10.1016/j.bjorl.2015.10.020 population. The lack of investment in preventive infrastructure has potentially great impact in populations at risk and this was clearly exemplified by the pandemic status that SARS-CoV2 achieved in 2020, in which many countries did not have even a straightforward policy on wearing masks and keeping physical distance, and did not have a clear message to achieve the $90 \%$ to $95 \%$ of compliance that would have stopped the cycle of transmissions.

\section{DATA AVAILABILITY STATEMENT}

The original contributions presented in the study are included in the article/supplementary material, further inquiries can be directed to the corresponding author/s.

\section{AUTHOR CONTRIBUTIONS}

AV conceptualized and wrote the essay. 
10. Vieira AR. Genetic and environmental factors in human cleft lip and palate. Front Oral Biol. (2012) 16:19-31. doi: 10.1159/000337521

11. Grant SF, Wang K, Zhang H, Glaberson W, Annaiah K, Kim CE, et al. A genome-wide association study identifies a locus for nonsyndromic cleft lip with or without cleft palate on 8q24. J Pediatr. (2009) 155:90913. doi: 10.1016/j.jpeds.2009.06.020

12. Mangold E, Ludwig KU, Birnbaum S, Baluardo C, Ferrian M, Herms S, et al. Genome-wide association study identifies two susceptibility loci for nonsyndromic cleft lip with or without cleft palate. Nat Genet. (2010) 42:246. doi: $10.1038 /$ ng.506

13. Beaty TH, Murray JC, Marazita ML, Munger RG, Ruczinski I, Hetmanski JB, et al. A genome-wide association study of cleft lip with and without cleft palate identifies risk variants near MAFB and ABCA4. Nat Genet. (2010) 42:525-9. doi: 10.1038/ng.580

14. Cavalcante BGN, Lacerda RHW, Assis IO, Bezamat M, Modesto A, Vieira AR. Talon cusp associates with MP2 in a cohort of individuals born with oral clefts. Cleft Palate Craniofac J. (2020) 58:597-602. doi: 10.1177/10556656209 58569

15. Van Dyck J, Llano-Pérula MC, Willems G, Verdonck A. Dental development in cleft lip and palate patients: a systematic review. Forensic Sci Int. (2019) 300:63-74. doi: 10.1016/j.forsciint.2019.04.011

16. Koruyucu M, Kasimoglu Y, Seymen F, Bayram M, Patir A, Ergöz N, et al. Rethinking isolated cleft lip and palate as a syndrome. Oral Surg Oral Med Oral Pathol Oral Radiol. (2018) 125:307-12. doi: 10.1016/j.oooo.2018. 01.007

17. Garland MA, Sun B, Zhang S, Reynolds K, Ji Y, Zhou CJ. Role of epigenetics and miRNAs in orofacial clefts. Birth Defect Res. (2020) 112:163559. doi: $10.1002 /$ bdr2.1802

18. Patel TA, Patel KG. Comparison of the Fisher anatomical subunit and modified Millard rotation-advancement cleft lip repairs. Plast Reconstr Surg. (2019) 144:2383-45e. doi: 10.1097/PRS.0000000000005847

19. Lacerda RHW, Vieira AR. Retrognathic maxillary in individuals born with oral clefts is due to the intrinsic factors of the cranial base and not only due to early surgical treatment. Angle Orthod. (2020) 91:24347. doi: 10.2319/060620-521.1

20. Hixon K, Rhea L, Standley J, Canady FJ, Canady JW, Dunnwald M. Interferon regulatory factor 6 controls proliferation of keratinocytes from children with Van der Woude syndrome. Cleft palate Craniofac J. (2017) 54:2816. doi: $10.1597 / 15-275$
21. Silva EMVM, Lacerda RHW, Farias IL, Cavalcante BGN, Assis IO, Bezamat $\mathrm{M}$, et al. COMT rs4818, pain sensitivity and duration, and alveolar bone grafting of oral clefts. Oral Maxillofac Surg. (2021) 25:2536. doi: 10.1007/s10006-020-00912-0

22. Winning L, Linden GJ. Periodontitis and systemic disease. BDJ Team. (2015) 2:15163. doi: 10.1038 /bdjteam.2015.163

23. Henn IW, Vieira AR. Worse caries experience does not lead to worse overall health. Pesqui Bras Odontopediatria Clin Integr. (2021) 21:e0028. doi: 10.1590/pboci.2021.094

24. Vieira AR. Periodontitis and Its Association with Systemic Diseases: Can Oral Bacteria Really Cause Other Diseases? (2020). Available online at: http://www. acbo.com.br/?page_id=6133 (accessed May 03, 2021).

25. Michalowicz BS, Hodges JS, DiAngelis AJ, Lupo VR, Novak MJ, Ferguson JE, et al. Treatment of periodontal disease and the risk of preterm birth. New Engl J Med. (2006) 355:1885-94. doi: 10.1056/NEJMoa062249

26. Zhao X, Liu Z, Shu D, Xiong Y, He M, Xu S, et al. Association of periodontitis with rheumatoid arthritis and the effect of non-surgical periodontal treatment on disease activity in patients with rheumatoid arthritis. Med Sci Monit. (2018) 24:5802-10. doi: 10.12659/MSM.909117

27. Mallick S, Benson R, Rath GK. Radiation induced oral mucositis: a review of current literature on prevention and management. Eur Arch Otorhinolaryngol. (2016) 273:2285-93. doi: 10.1007/s004405-015-3694-6

28. Vieira AR. Genetic basis of lichen planus and oral cancer. In: Vieira AR, editor. Genetic Basis of oral Health Conditions. Cham: Springer (2019). p. 73-9.

29. Sharbaugh MS, Althouse AD, Thoma FW, Lee JS, Figueiredo VM, Mulukutla SR. Impact of cigarette taxes on smoking prevalence from 2001-2015: a report using the Behavioral and Risk Factor Surveillance Survey (BRFSS). PLoS ONE. (2018) 13:3020416. doi: 10.1371/journal.pone.0204416

Conflict of Interest: The author declares that the research was conducted in the absence of any commercial or financial relationships that could be construed as a potential conflict of interest.

Copyright $\odot 2021$ Vieira. This is an open-access article distributed under the terms of the Creative Commons Attribution License (CC BY). The use, distribution or reproduction in other forums is permitted, provided the original author(s) and the copyright owner(s) are credited and that the original publication in this journal is cited, in accordance with accepted academic practice. No use, distribution or reproduction is permitted which does not comply with these terms. 